\title{
Evidence of Fermi surface reconstruction at the metamagnetic transition of the strongly correlated superconductor $\mathrm{UTe}_{2}$
}

\author{
Q. Niu $\odot,{ }^{1}$ G. Knebel $\odot,{ }^{1}$ D. Braithwaite $\odot,{ }^{1}$ D. Aoki,,${ }^{1,2}$ G. Lapertot, ${ }^{1}$ M. Vališka $\odot,{ }^{1}$ G. Seyfarth $\odot,{ }^{3}$ W. Knafo $\odot,{ }^{4}$ \\ T. Helm $\odot{ }^{5,6}$ J.-P. Brison ${ }^{\circ},{ }^{1}$ J. Flouquet, ${ }^{1}$ and A. Pourret ${ }^{10}{ }^{1, *}$ \\ ${ }^{1}$ Université Grenoble Alpes, CEA, IRIG, PHELIQS, F-38000 Grenoble, France \\ ${ }^{2}$ Institute for Materials Research, Tohoku University, Oarai, Ibaraki 311-1313, Japan \\ ${ }^{3}$ Université Grenoble Alpes, EMFL, CNRS, Laboratoire National des Champs Magnétiques Intenses (LNCMI), \\ 38042 Grenoble, France \\ ${ }^{4}$ Laboratoire National des Champs Magnétiques Intenses, UPR 3228, CNRS-UPS-INSA-UGA,143 Avenue de Rangueil, \\ 31400 Toulouse, France \\ ${ }^{5}$ Max Planck Institute for Chemical Physics of Solids, 01187 Dresden, Germany \\ ${ }^{6}$ Dresden High Magnetic Field Laboratory (HLD-EMFL), Helmholtz-Zentrum Dresden-Rossendorf, 01328 Dresden, Germany
}

(Received 19 March 2020; accepted 16 June 2020; published 3 August 2020)

\begin{abstract}
Thermoelectric power $(S)$ and Hall effect $\left(R_{\mathrm{H}}\right)$ measurements on the paramagnetic superconductor $\mathrm{UTe}_{2}$ with the magnetic field applied along the hard magnetization $b$ axis are reported. The first-order nature of the metamagnetic transition at $H_{\mathrm{m}}=H_{\mathrm{c} 2}^{b}=35 \mathrm{~T}$ leads to drastic consequences on $S$ and $R_{\mathrm{H}}$. In contrast to the field dependence of the specific heat in the normal state through $H_{\mathrm{m}}, S(H)$ is not symmetric with respect to $H_{\mathrm{m}}$. This implies a strong interplay between ferromagnetic fluctuations and a Fermi-surface reconstruction at $H_{\mathrm{m}}$. $R_{\mathrm{H}}$ is very well described by incoherent skew scattering above the coherence temperature $T_{\mathrm{m}}$ corresponding roughly to the temperature of the maximum in the susceptibility $T_{\chi_{\max }}$ and coherent skew scattering at lower temperatures. The discontinuous field dependence of both $S(H)$ and the ordinary Hall coefficient $R_{0}$, at $H_{\mathrm{m}}$ and at low temperature, provides evidence of a change in the band structure at the Fermi level.
\end{abstract}

DOI: 10.1103/PhysRevResearch.2.033179

\section{INTRODUCTION}

The recent discovery of unconventional superconductivity (SC) in the uranium chalcogenide paramagnet $\mathrm{UTe}_{2}$ with a superconducting transition temperature of $T_{\mathrm{SC}} \sim 1.6 \mathrm{~K}$ [1-3] opens new perspectives on superconducting topological properties including emergent Majorana quasiparticles at the verge of magnetic and electronic instability. Transport and thermodynamic measurements demonstrated that correlations play an important role in this system, requiring theoretical treatment beyond the local-density approximation approach [2,48]. The closeness of $\mathrm{UTe}_{2}$ to ferromagnetic quantum criticality [9] induces astonishing superconducting properties. Indeed when the magnetic field is applied along the hard $b$ axis at low temperature, superconductivity survives up to an extremely high field, $H_{\mathrm{c} 2}=35 \mathrm{~T}$, where it is destroyed abruptly by the occurrence of a huge metamagnetic transition at $H_{\mathrm{m}}=H_{\mathrm{c} 2}[3,10]$. The unconventional superconducting state in this system, i.e., spin-triplet Cooper pairing, has been identified by a small decrease in the NMR Knight

\footnotetext{
*alexandre.pourret@cea.fr

Published by the American Physical Society under the terms of the Creative Commons Attribution 4.0 International license. Further distribution of this work must maintain attribution to the author(s) and the published article's title, journal citation, and DOI.
}

shift [11] and the large $H_{\mathrm{c} 2}$ exceeding the Pauli-limiting field [1-3]. Furthermore, reentrant superconductivity arises above $H_{\mathrm{m}}$ when the magnetic field is tilted $30^{\circ}$ away from the $b$ axis towards the $c$ axis [10]. The metamagnetic transition occurring at $H_{\mathrm{m}}$ with a jump in the magnetization of $0.6 \mu_{B}$, when the system enters the polarized paramagnetic state (PPM) $[10,12,13]$, is in agreement with a characteristic energy scale given by the temperature of the maximum in the susceptibility of $T_{\chi_{\max }} \approx 35 \mathrm{~K}$ [14] and the maximum of the Hall effect $\left(R_{\mathrm{H}}\right)$ [4]. Furthermore, fluctuations are strongly enhanced through $H_{\mathrm{m}}$ despite the first-order nature of the metamagnetic transition below the critical end point (CEP) at $T_{\mathrm{CEP}} \approx 7 \mathrm{~K}[12,14]$.

By some aspects, $\mathrm{UTe}_{2}$ has properties similar to those found in the unconventional ferromagnetic superconductor URhGe [15]. It shows similar field enhancement of the Sommerfield coefficient $\gamma$ (linear $T$ term of the specific heat) associated with reentrant superconductivity when approaching $H_{\mathrm{m}}\left(H_{\mathrm{m}}=H_{\mathrm{r}} \approx 11.75 \mathrm{~T}\right.$ in URhGe) [16-18]. In URhGe, the metamagnetic transition is connected to a Fermi-surface instability $[19,20]$ which may drive the SC [21,22]. In $\mathrm{UTe}_{2}$ as well as in URhGe, the metamagnetic transition occurs for the field along the hard magnetization axis. In both systems the metamagnetic transition is strongly connected to the field enhancement of the SC. A major difference is that $\mathrm{URhGe}$ is ferromagnetic with a Curie temperature of $T_{\mathrm{C}}=9.5 \mathrm{~K}$ at $H=0$ while $\mathrm{UTe}_{2}$ remains paramagnetic (PM) at least down to $20 \mathrm{mK}[23,24]$. 
A key question is the respective roles of ferromagnetic fluctuations and Fermi-surface instabilities at the metamagnetic transition where the SC is abruptly suppressed. Indeed, the large steplike increase of the residual term of the resistivity, $\rho_{0}$, at the metamagnetic transition suggests that, in addition to magnetic fluctuations, a change in the carrier density may occur at the metamagnetic transition for $H \| b$ [14]. For this purpose, we investigated the temperature and magnetic field dependencies of the Seebeck coefficient $(S)$ up to $36 \mathrm{~T}$ and the Hall resistance $\left(R_{\mathrm{H}}\right)$ up to $68 \mathrm{~T}$ of $\mathrm{UTe}_{2}$ for $H \| b$. Reentrant superconductivity is observed in both $S$ and $R_{\mathrm{H}}$ close to $H_{\mathrm{m}}$ around $1 \mathrm{~K}$, consistent with resistivity results [3]. The drastic changes in $S$ and in the ordinary Hall effect $\left(R_{0}\right)$ at $H_{\mathrm{m}}$ point to a Fermi-surface reconstruction, contrasting with the rather symmetric behavior of the $\gamma$ term [12] and of the $A$ coefficient (the $T^{2}$ term of the resistivity) through $H_{\mathrm{m}}$ [14].

\section{EXPERIMENTAL DETAILS}

Single crystals of $\mathrm{UTe}_{2}$ were grown by chemical vapor transport with iodine as the transport agent. The orientation of the crystals has been verified by Laue diffraction. We performed the $S, \rho$, and $R_{\mathrm{H}}$ measurements on three samples labeled S1, S2, and $\mathrm{S} 3$ with a residual resistivity ratio $\left(\mathrm{RRR}=\frac{\rho(300 \mathrm{~K})}{\rho(1.5 \mathrm{~K})}\right)$ of 30,30 , and 22 , respectively. The samples were prepared for experiments with heat or electric current along the $a$ axis and the magnetic field along the $b$ axis. $S$ and $R_{\mathrm{H}}$ have been measured on sample $\mathrm{S} 1$ using a standard "one heater-two thermometers" setup, and $\rho_{x x}$ and $R_{\mathrm{H}}$ have been measured on samples S2 and S3 with a standard six-point method. The temperature and field dependencies of different transport properties have been measured at the LNCMI Grenoble using a ${ }^{3} \mathrm{He}$ cryostat up to $36 \mathrm{~T}$ and on sample $\mathrm{S} 3$ at the LNCMI Toulouse in a pulsed field up to $68 \mathrm{~T}$ and temperatures down to $1.5 \mathrm{~K}$.

\section{RESULTS AND DISCUSSION}

\section{A. Thermoelectric power}

Figure 1 shows the magnetic field dependence of $S$ from 0 to $36 \mathrm{~T}$ [panel (a)] and from 32 to $36 \mathrm{~T}$ [panels (b) and (c)] at various temperatures. At $0.7 \mathrm{~K}, S$ is equal to zero up to the first-order transition at $H_{\mathrm{m}}=34.6 \mathrm{~T}$, where $S$ shows a clear negative jump followed by a rapid increase in agreement with the collapse of SC above $H_{\mathrm{m}}$. At the slightly higher temperature $0.86 \mathrm{~K}$, the sample enters the normal state at about $13 \mathrm{~T}$ with a negative $S$, as indicated by the arrows in the inset of Fig. 1(a). A field-induced reentrant superconductivity phase is then observed between 27 and $34.6 \mathrm{~T}$. The first-order character of the transition is also observed in our $S(H)$ measurements with a strong hysteresis (see Fig. 2). Upon warming, the hysteresis closes and the jump vanishes, indicating that the first-order transition terminates at a CEP with $T_{\mathrm{CEP}} \approx 7 \mathrm{~K}$ in agreement with previous measurements [12,14]. Below $T_{\mathrm{CEP}}$, there is a slight increase of $|S(H)|$ on approaching $H_{\mathrm{m}}$, and then $S(H)$ changes abruptly at $H_{\mathrm{m}}$. Interestingly, the negative jump at $H_{\mathrm{m}}$ disappears at $3.4 \mathrm{~K}$ and it becomes positive at higher temperatures, as shown more clearly in Fig. 3(a), where we plot $\Delta S\left(H_{\mathrm{m}}\right) / T$ as a function of temperature up to $5 \mathrm{~K}$. The amplitude of the
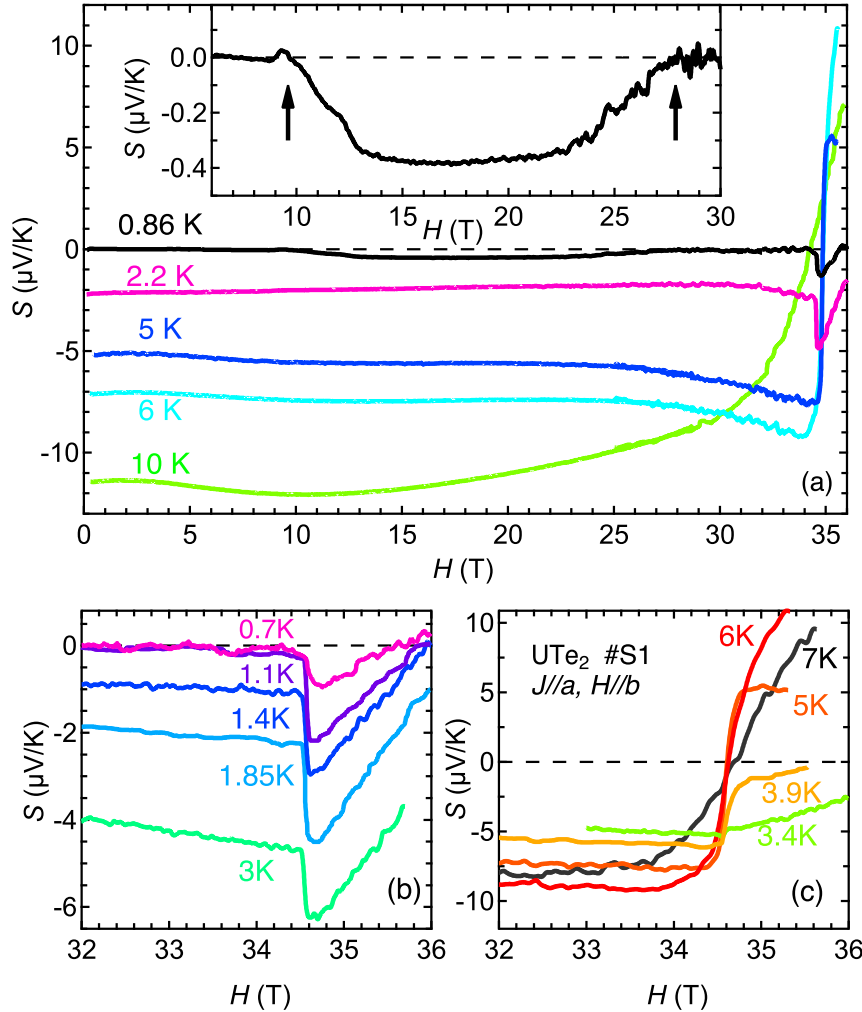

FIG. 1. Field dependence of $S$ in $\mathrm{UTe}_{2}$ for $H \| b$ between 0 and $36 \mathrm{~T}$ (a) and between 32 and $36 \mathrm{~T}(\mathrm{~b}, \mathrm{c}) . S(H)$ shows a clear anomaly at $H_{\mathrm{m}}=34.6 \mathrm{~T}$, which broadens with increasing temperature. This anomaly changes from a negative to a positive jump above $3.5 \mathrm{~K}$ The inset in panel (a) shows the reentrant superconductivity in $S(H)$ at $0.86 \mathrm{~K}$ in the field range from 6 to $30 \mathrm{~T}$.

transition increases up to $\approx 3 \mu \mathrm{V} / \mathrm{K}^{2}$ at $5 \mathrm{~K}$. For $T=10 \mathrm{~K}$, above $T_{\mathrm{CEP}}$ only a large crossover can be detected.

Figure 3(b) shows the temperature dependence of $S(T) / T$ between 0 and $7 \mathrm{~K}$ for different magnetic fields. For $H<H_{\mathrm{m}}$ (solid symbols), $S(T) / T \approx 1 \mu \mathrm{V} / \mathrm{K}^{2}$ is temperature independent in the normal state in this temperature range and for a

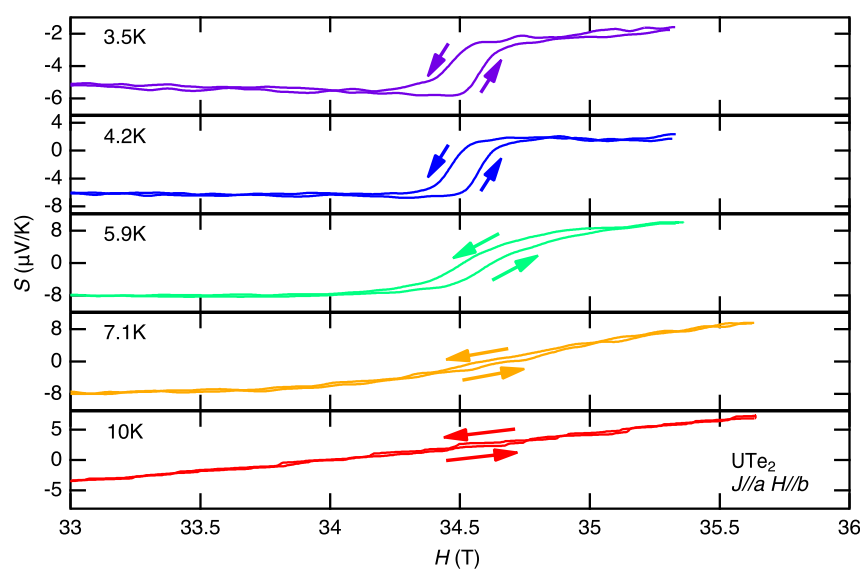

FIG. 2. Temperature evolution of the hysteresis observed in $S$ at $H_{\mathrm{m}}$. The arrows indicate the direction of the field sweep. The firstorder transition ends at the critical end point $T_{\mathrm{CEP}} \approx 7 \mathrm{~K}$. 

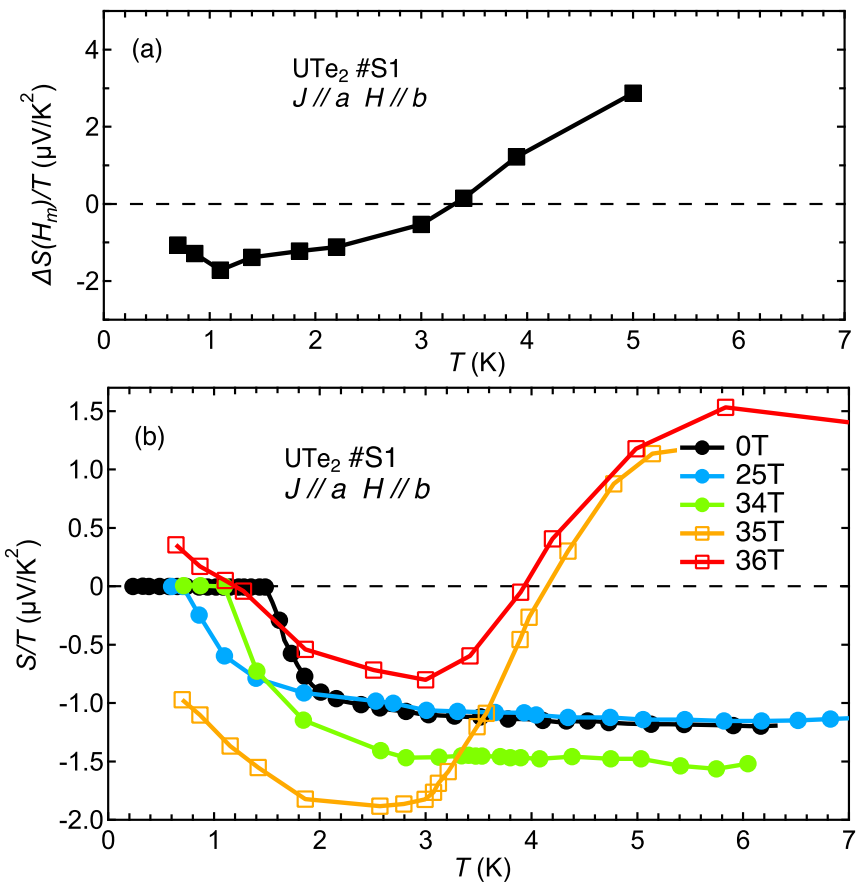

FIG. 3. (a) Magnitude of the jump $\Delta S / T=\frac{S\left(H_{\mathrm{m}}+\delta H\right)-S\left(H_{\mathrm{m}}-\delta H\right)}{T}$ in $S / T$ at $H_{\mathrm{m}}$. (b) Temperature dependence of $S / T$ at different magnetic fields (solid symbols, $H<H_{m}$; open symbols, $H>H_{m}$ ). $S / T$ changes drastically with temperature near $H_{\mathrm{m}}$.

field below $24 \mathrm{~T}$. $|S(T) / T|$ is slightly larger on approaching $H_{\mathrm{m}}$ as shown for $34 \mathrm{~T}$. In contrast, $S(T) / T$ displays a very different temperature dependence above $H_{\mathrm{m}}$ (open symbols). For instance, at $35 \mathrm{~T}, S / T(T)<0$ at low temperature (SC is suppressed) and decreases up to $3 \mathrm{~K}$. Above $3 \mathrm{~K}$, it increases drastically and changes sign at around $4.1 \mathrm{~K}$. Moreover, at $36 \mathrm{~T}$, the interesting feature is that $S / T$ becomes positive at very low temperature below $1.4 \mathrm{~K}$. This shows that at low temperature the sign of the dominant heat carriers changes through $H_{\mathrm{m}}$ from electrons to holes.

\section{B. Hall effect}

To extract more information about the field dependence of the carriers across $H_{\mathrm{m}}$, we measured the Hall effect up to $36 \mathrm{~T}$ on sample $\mathrm{S} 2$ in the static field and up to $68 \mathrm{~T}$ on sample $\mathrm{S} 3$ in the pulsed field. Figures 4(a) and 4(b) show the field dependence of the Hall resistivity $\rho_{x y}$ measured on S2 at different temperatures. At $0.45 \mathrm{~K}$, the sample is superconducting up to the metamagnetic transition. Above $H_{\mathrm{m}}, \rho_{x y}$ is positive in the normal state. At $1 \mathrm{~K}$, reentrant superconductivity is detected in $\rho_{x y}$ as indicated by the arrows. However, a negative $\rho_{x y}$ shows up in the normal state below the reentrant superconductivity. As the temperature increases, the transition in $\rho_{x y}$ at $H_{\mathrm{m}}$ becomes huge with a maximum value at $7.5 \mathrm{~K}$ near the CEP. At the same time, the initial slope of $\rho_{x y}(H)$ at low field also increases rapidly from negative to positive. The inset of Fig. 4(a) illustrates the temperature dependence of the Hall coefficient $R_{\mathrm{H}}=\rho_{x y} / H$ at different fields. At $9 \mathrm{~T}$, where $\rho_{x y}(H)$ is still linear, $R_{\mathrm{H}}(T)$ changes rapidly from negative

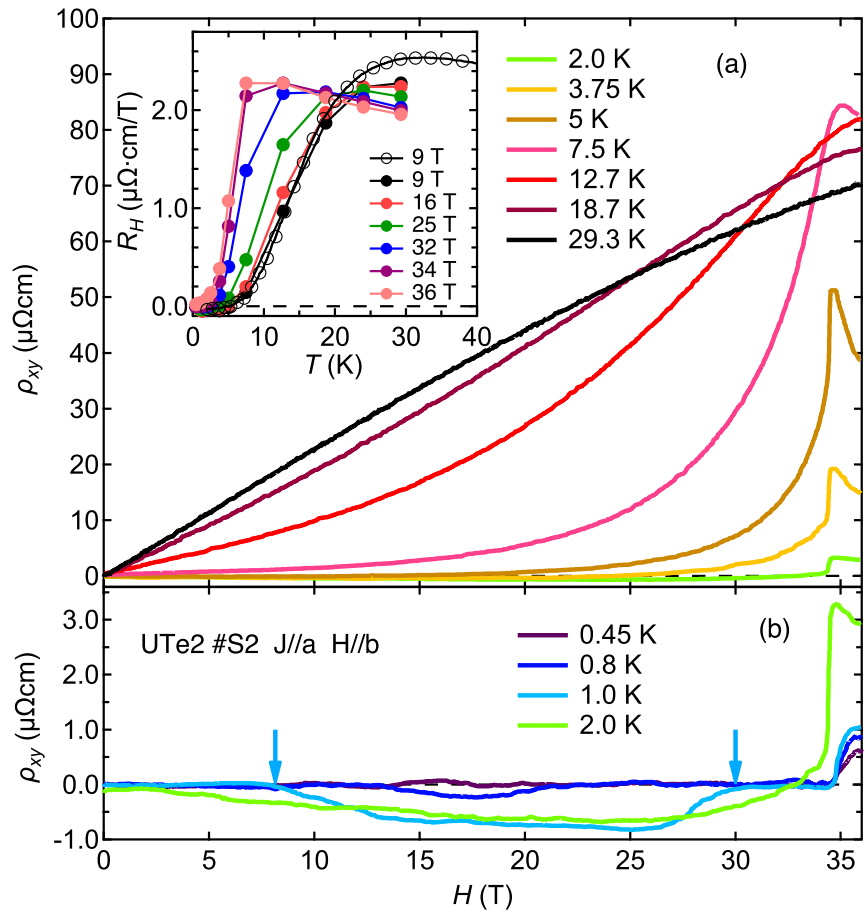

FIG. 4. (a) The Hall resistivity of $\mathrm{UTe}_{2}$ with $H \| b$ up to $36 \mathrm{~T}$ at different temperatures. Panel (b) shows a zoom on low temperatures. The arrows indicate the normal state and reentrant superconductivity at $1 \mathrm{~K}$. The inset of panel (a) shows the $T$ dependence of the Hall coefficient at different fields. Previous data are also represented (open circles) [4].

to positive and shows a maximum at $T_{\mathrm{m}} \approx 30 \mathrm{~K}$ close to $T_{\chi_{\max }}$ [4]. This drastic increase of the Hall coefficient at low temperature, which has been observed in many heavy fermion systems, like $\mathrm{UPt}_{3}[25]$ and $\mathrm{UAl}_{2}$ [26], is related to the change of the scattering process from incoherent skew scattering at high temperature to a coherent scattering regime at low temperature [27]. As $H$ increases, $R_{\mathrm{H}}(T)$ becomes steeper and $T_{\mathrm{m}}$ shifts to lower temperature until the metamagnetic transition at $H_{\mathrm{m}}$, where $T_{\mathrm{m}}$ ends at about $7 \mathrm{~K}$ close to the CEP (see also Fig. 6). Above $H_{\mathrm{m}}, R_{\mathrm{H}}$ decreases with the field and the temperature where $R_{\mathrm{H}}$ is maximum (labeled $T_{\mathrm{cr}}$ ) indicates the PM-PPM crossover $[19,28]$. It shifts to higher temperature when increasing the magnetic field, see also Fig. 9.

In the presence of magnetic fluctuations, $R_{\mathrm{H}}$ can be described by the sum of an ordinary part, $R_{0}$, and an anomalous part, $R_{\mathrm{S}} . R_{0}$ is simply related to the density and the mobility of the carriers, while $R_{\mathrm{S}}$ is the result of different scattering processes. In heavy-fermion systems, the incoherent skew scattering of conduction electrons by independent local $f$ moments predominates at high temperature above the coherence temperature [29]. $R_{\mathrm{S}}$ is proportional to the magnetic susceptibility $\chi$ and the electrical resistivity $\rho_{x x}$, i.e., $R_{\mathrm{S}} \propto$ $\rho_{x x} \chi$. This has been verified in many materials in the hightemperature incoherent regime. When the coherence settles in at low temperature, a different scattering mechanism, $R_{\mathrm{S}} \propto$ $\rho_{x x}^{2} \chi$, has been observed in many uranium heavy-fermion compounds [26], and this is theoretically explained by coherent skew scattering [30]. 

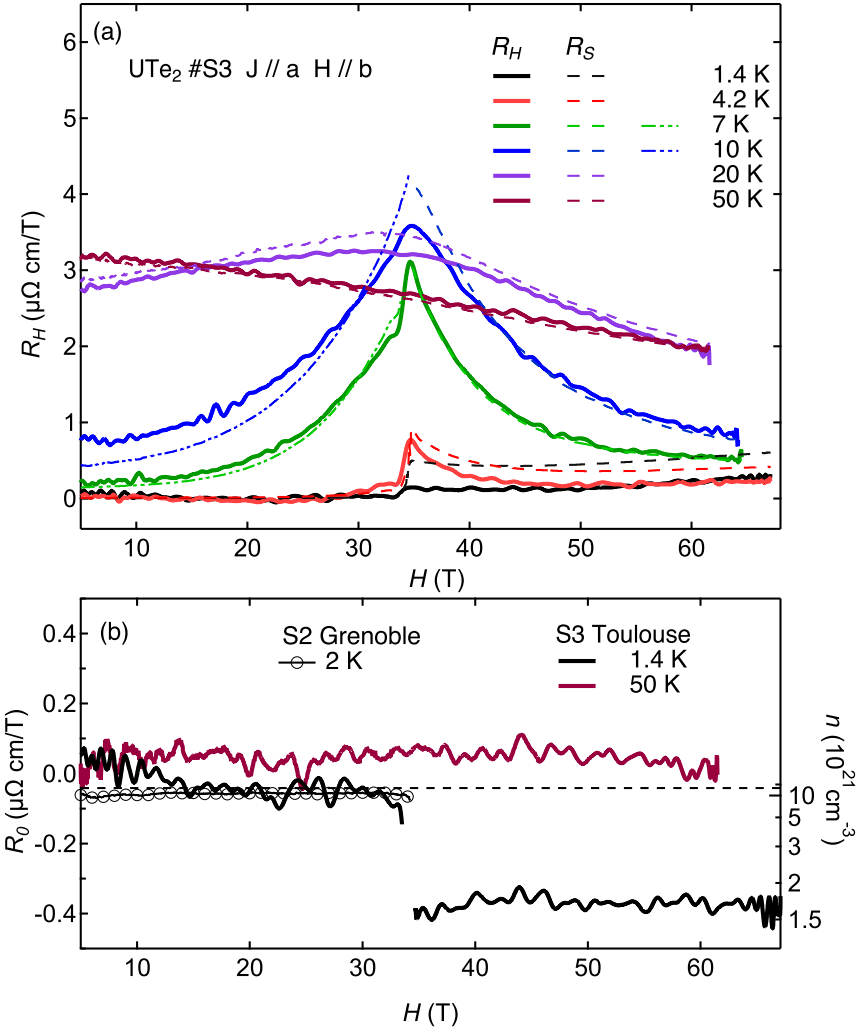

FIG. 5. (a) The Hall coefficient of $\mathrm{UTe}_{2} \mathrm{~S} 3$ with $H \| b$ up to $68 \mathrm{~T}$ (pulsed field). The dashed lines are estimated from the coherent $(T<20 \mathrm{~K})$ and incoherent $(T \geqslant 20 \mathrm{~K})$ skew scattering at different temperatures. For $T<20 \mathrm{~K}$, the long and short dashes correspond to the anomalous Hall signal obtained above and below $H_{\mathrm{m}}$, respectively. (b) $R_{0}$ is obtained after subtracting the anomalous part from the Hall signal (additional temperatures are shown in Fig. 8). The right scale indicates the carrier density and the dashed line represents the value obtained previously [4].

In order to get information on the change of carriers in $\mathrm{UTe}_{2}$ (for details, see the Appendix), we have used the magnetization data of $\mathrm{UTe}_{2}$ from Ref. [12] and plotted $R_{x y} / H$ against $R_{x x} M / H$ or $R_{x x}^{2} M / H$ at different temperatures (see Figs. 11,12 and 13). $R_{x y} / H$ is linear against $R_{x x}^{2} M / H$ up to $\sim 10 \mathrm{~K}$. However, the curves below and above $H_{\mathrm{m}}$ fall onto two different lines with different slopes and/or intercepts, indicating that both $R_{0}$ and $R_{\mathrm{S}}$ have discontinuous changes at $H_{\mathrm{m}}$. In contrast, at $50 \mathrm{~K}$, which is above $T_{\mathrm{m}}$, the coherence temperature, $R_{x y} / H$ is on a straight line with $R_{x x} M / H$ in almost the whole field range, consistent with the incoherent skew scattering predictions. The analysis shows that the Hall effect below $10 \mathrm{~K}$ is dominated by coherent skew scattering, and the Hall effect above $10 \mathrm{~K}$ by incoherent skew scattering. This allows us to estimate the contribution of $R_{0}$ to the total Hall effect. The solid lines in Fig. 5(a) show $R_{x y} / H$, while the dashed lines correspond to the anomalous Hall contribution obtained from the fitting by considering the change of slope of the anomalous Hall effect below (short dash) and above (long dash) $H_{\mathrm{m}}$. The difference of these two datasets gives an estimation of $R_{0}$ as plotted in Fig. $5(\mathrm{~b})$. At $1.4 \mathrm{~K}$, below $H_{\mathrm{m}}$, $R_{0}$ reflects the fact that the anomalous Hall effect vanishes at

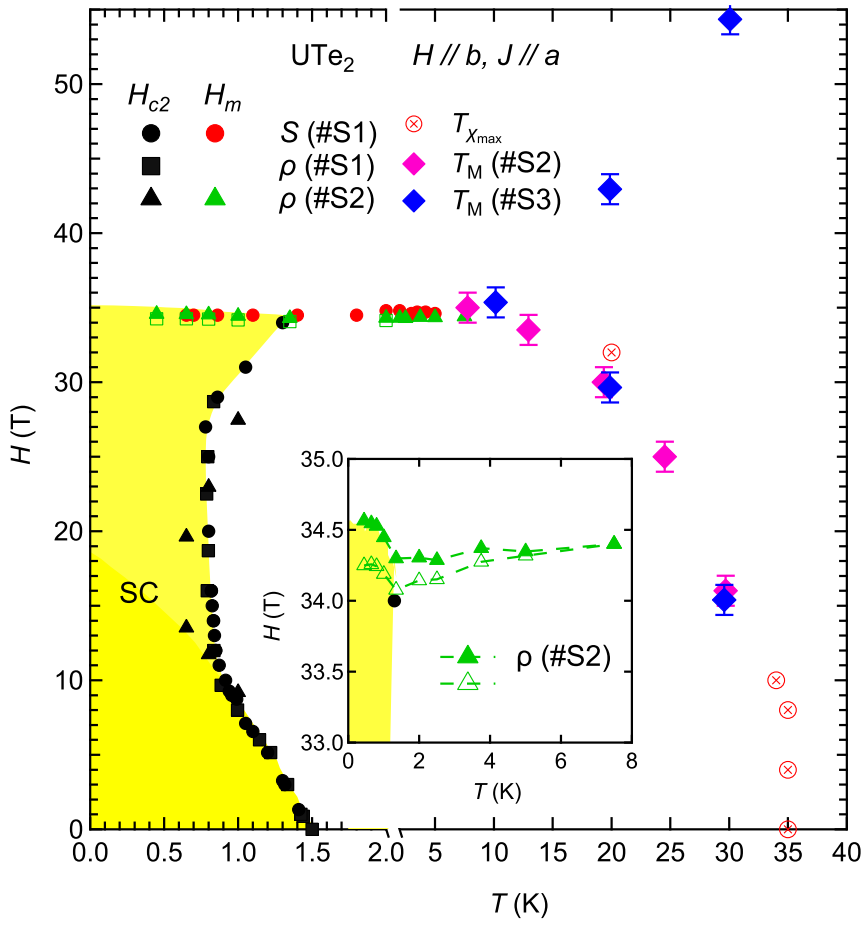

FIG. 6. $H-T$ phase diagram of $\mathrm{UTe}_{2}$ for $H \| b . H_{\mathrm{c} 2}$ from different samples is shown by black symbols, and the metamagnetic field $H_{\mathrm{m}}$ is labeled by red circles $(S)$ and red triangles $(\rho)$. Red crosses, pink diamonds, and blue diamonds indicate $T_{\chi_{\max }}, T_{\mathrm{m}}$, and $T_{\mathrm{cr}}$, respectively. The inset shows a zoom very close to $H_{\mathrm{m}}$. Solid symbols are from increasing field sweeps, and open symbols are from downward field sweeps. SC survives slightly above the metamagnetic transition.

low temperature (see also Fig. 10). $R_{0}$ is negative, very small, and independent of the magnetic field up to $H_{\mathrm{m}}$. The value of the extracted carrier density (right scale) is in good agreement with the value obtained previously (dashed line) [4], $n=$ $1.6 \times 10^{22} \mathrm{~cm}^{-3}$. Above $H_{\mathrm{m}},\left|R_{0}\right|$ is much larger and still field independent. Most likely, such a behavior is the signature of a change of the carrier density (accompanied or not with a change of the mobility) at the metamagnetic transition. In contrast, at $50 \mathrm{~K}$ entering into the incoherent regime, the Hall coefficient can be very well reproduced by anomalous Hall terms.

The upper critical field $H_{\mathrm{c} 2}(T)$ and $H_{\mathrm{m}}$ detected in $S$ and $\rho$ are summarized in the $H-T$ phase diagram in Fig. 6. $H_{\mathrm{c} 2}(T)$ is defined by $S=0$ or $\rho=0$. Above $10 \mathrm{~T}, S$ and $\rho$ show an almost vertical $H_{\mathrm{c} 2}(T)$ between 10 and $28 \mathrm{~T}$, and $H_{\mathrm{c} 2}$ is strongly enhanced, reaching $1.4 \mathrm{~K}$ at $H_{\mathrm{m}}$, consistent with previous resistivity experiments $[1,3]$. The temperatures of the maximum of the Hall effect, $T_{\mathrm{m}}$ (similar to $T_{\chi_{\max }}$ ), and $T_{\mathrm{cr}}$ are also represented. This is similar to the energy scales observed near the tricritical point in the ferromagnetic superconductor URhGe with the same crossover line separating PM and PPM states [19]. Similar thermoelectric power experiments in the nearly ferromagnetic (FM) case of UCoAl [31] have identified the CEP of the first-order transition at the metamagnetic transition from PM to FM states. This material is an itinerant Ising system, where the metamagnetic transition occurs with $H$ along the Ising axis. 

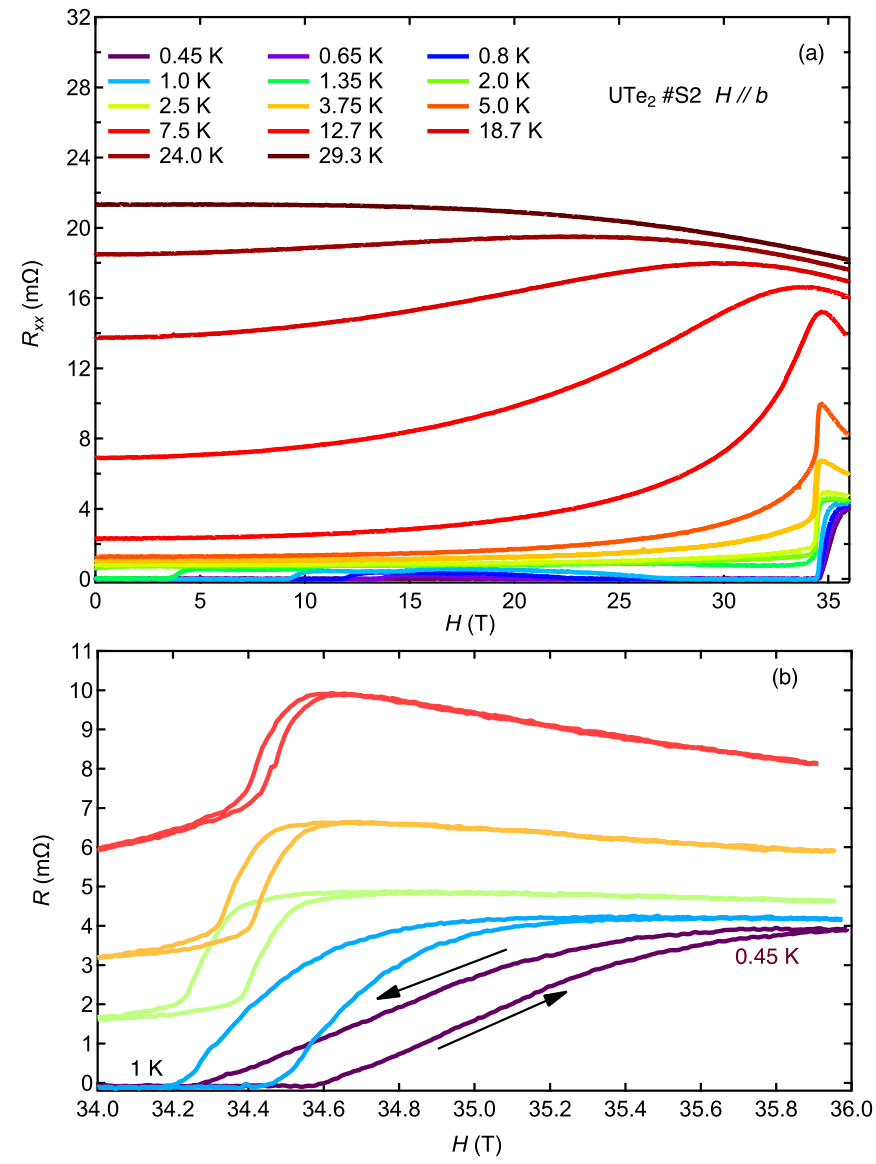

FIG. 7. (a) Field dependence of $R_{x x}$ on sample $\mathrm{S} 2$ up to $36 \mathrm{~T}$ at different temperatures for $H \| b$. (b) Hysteresis loop of $R_{x x}$ at the metamagnetic transition highlighting the first-order character of the transition below $T_{\mathrm{CEP}} \approx 7 \mathrm{~K}$.

In contrast, in $\mathrm{UTe}_{2}$ as well as in URhGe, the metamagnetic transition occurs for the field along the hard magnetization axis. In both systems the metamagnetic transition is strongly connected to the field enhancement of the SC. The inset magnifies the phase diagram near the metamagnetic transition transition. We observe that $H_{\mathrm{m}}\left(H_{\mathrm{c} 2}\right)$ has an upturn below $1 \mathrm{~K}$. This feature indicates that SC persists above the extrapolation of $H_{\mathrm{m}}$ to $T=0 \mathrm{~K}$, although in a very narrow field range.

\section{CONCLUSION}

In conclusion, we have studied the thermoelectric power and the Hall effect of the PM superconductor $\mathrm{UTe}_{2}$ up to 36 and $68 \mathrm{~T}$, respectively, with the magnetic field along the hard magnetization $b$ axis. Reentrant superconductivity was observed in both $S$ and $R_{\mathrm{H}}$ at low temperature on approaching the metamagnetic transition. $R_{\mathrm{H}}$ is very well described by incoherent skew scattering above the coherence temperature $T_{M}$, which corresponds roughly to $T_{\chi_{\max }}$, and by coherent skew scattering at lower temperatures. The correspondence of these two energy scales highlights the dual character, localizeditinerant, of the $f$ electrons in uranium compounds. Below this Kondo coherence temperature, density functional theory calculations reveal the emergence of a band structure with a small

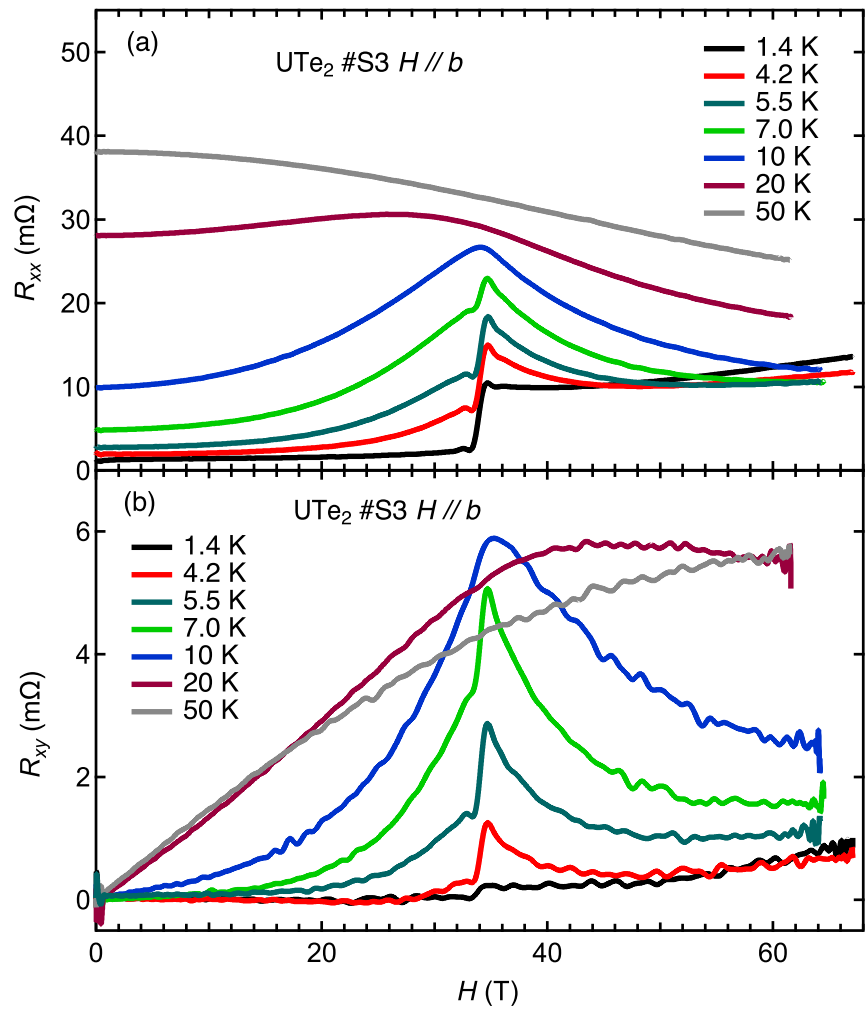

FIG. 8. Field dependence of $R_{x x}$ (a) and $R_{x y}$ (b) on sample S3 up to $68 \mathrm{~T}$ at different temperatures.

peak in the density of states at the Fermi level [8]. The field dependence of $R_{\mathrm{H}}$ suggests the suppression of well-defined coherent scattering by strong magnetic fluctuations near the metamagnetic transition. Above $T_{\mathrm{SC}}$, for $H<H_{\mathrm{m}}$, the anomalous part of the Hall signal vanishes, opposite to the case $H>H_{\mathrm{m}}$, where the anomalous part is still present. The strong change of $R_{0}$ and $S$ evidences a Fermi-surface reconstruction

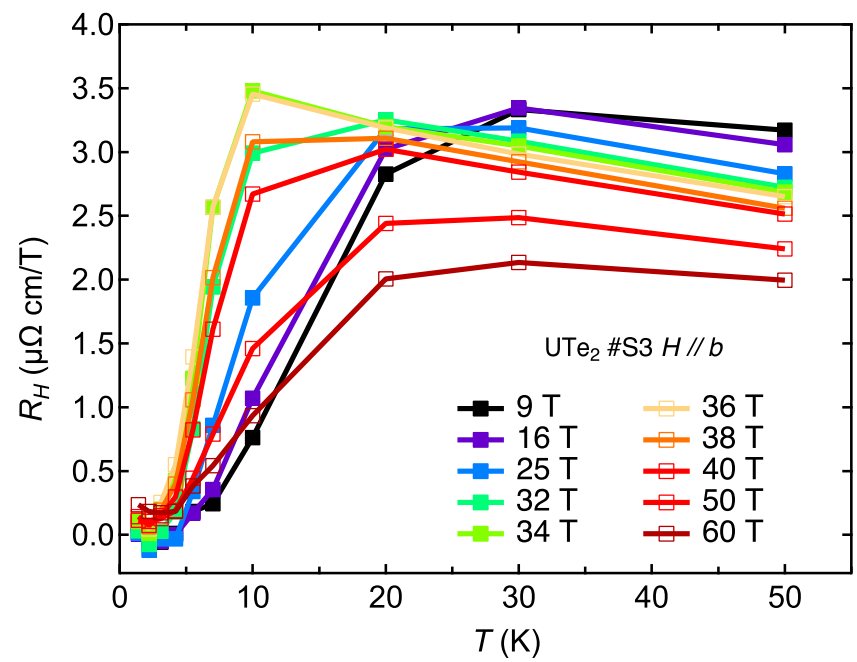

FIG. 9. Temperature dependence of $R_{\mathrm{H}}$ on sample $\mathrm{S} 3$ at different magnetic fields for $H<H_{\mathrm{m}}$ (solid symbols) and for $H>H_{\mathrm{m}}$ (open symbols). 


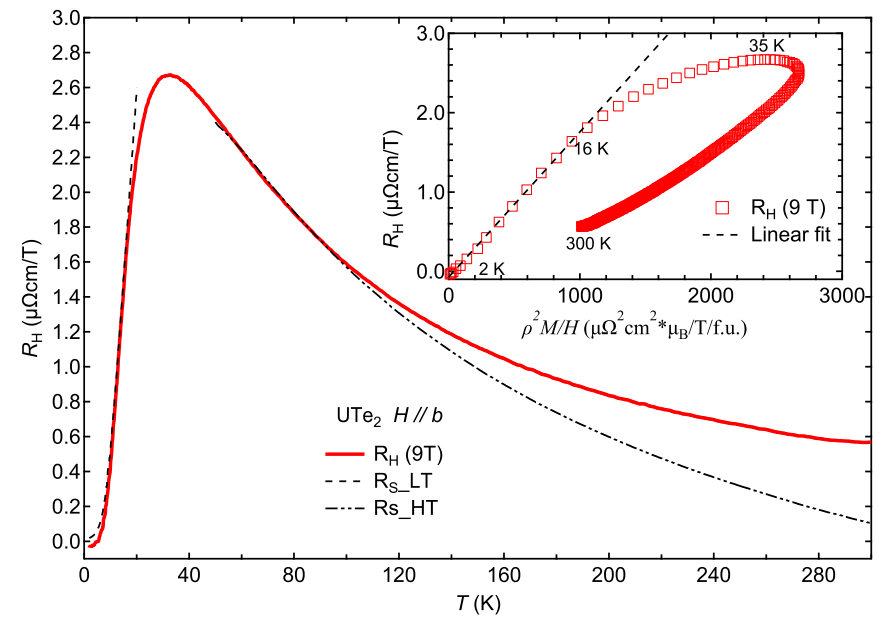

FIG. 10. Temperature dependence of $R_{\mathrm{H}}$ between 1.4 and $300 \mathrm{~K}$. The different fitting curves for the anomalous Hall effect at low temperature $\left(\propto \rho^{2} M / H\right)$ and high temperature $(\propto \rho M / H)$ are represented (dashed lines). Inset: $R_{\mathrm{H}}$ as a function of $\rho^{2} M / H$. The dashed line represents linear fitting.

at $H_{\mathrm{m}}$. As this Fermi surface change leads to the vanishing of SC above $H_{\mathrm{m}}$, a drastic change in the nature of the SC pairing may happen, contrasting with a rather symmetrical variation of $\gamma(H)$ on crossing $H_{\mathrm{m}}$. A still open question is the anomalous SC phase which persists at $30^{\circ}$ from the $b$ axis in the PPM state above $H_{\mathrm{m}}$. The originality of $\mathrm{UTe}_{2}$ consists in its proximity to a Kondo-lattice metal-insulator instability which deserves to be studied further under both magnetic field and pressure.

\section{ACKNOWLEDGMENTS}

The authors thank K. Izawa, S. Hosoi, H. Harima, J. Ishizuka, and Y. Yanase for stimulating discussions. We acknowledge A. Miyake for high magnetic field magnetization data. This work has been supported by the Université Grenoble Alpes IRS project and KAKENHI (Grants No. JP15H0082, No. JP15H0084, No. JP15K21732, No. JP19H00646, No. JP16H04006, and No. JP15H05745). We acknowledge support of the LNCMI-CNRS, member the European Magnetic Field Laboratory (EMFL).

\section{APPENDIX}

\section{Resistivity and Hall signal for $H \| b$}

The resistivity and the Hall effect have been measured on samples S2 and S3, part of the results have been already shown in the main text. Figure 7(a) shows the magnetic field dependence of $R_{x x}$ for different temperatures up to $36 \mathrm{~T}$ for sample S2. Below $1.5 \mathrm{~K}, R_{x x}$ is zero up to $H_{\mathrm{m}}$ and then shows a steplike anomaly indicating that $H_{\mathrm{c} 2}$ is equal to $H_{\mathrm{m}}$ for $H \| b$. By increasing temperature, $R_{x x}$ is nonzero below $H_{\mathrm{m}}$ but still shows a steplike anomaly at the metamagnetic transition up to $T_{\mathrm{CEP}} \approx 7 \mathrm{~K}$. Above $T_{\mathrm{CEP}}$, the transition broadens significantly. The first-order character of the transition below $T_{\mathrm{CEP}}$ is also confirmed by the observation of a hysteresis loop in the resistivity [see Fig. 7(b)]. Interestingly, as soon as the system becomes superconducting below $1.5 \mathrm{~K}$, the width of the transition broadens and the superconducting transition seems to move to a higher field, conserving a hysteresis loop.

The field dependence of $R_{x x}$ and $R_{x y}$ up to $68 \mathrm{~T}$ of sample $\mathrm{S} 3$ are represented in Fig. 8. The resistivity data are similar to sample S2. At low temperature, $R_{x y}$ is almost field independent below $H_{\mathrm{m}}$, shows a small step like anomaly at $H_{\mathrm{m}}$, and becomes field dependent above $H_{\mathrm{m}}$. By increasing the temperature, the transition becomes a peak which is very sharp at $T_{\mathrm{CEP}} \approx 7 \mathrm{~K}$ and broadens for high temperature. Figure 9 shows the temperature dependence of the Hall coefficient $R_{\mathrm{H}}$ measured on sample S3. Starting from low-magnetic field, the temperature of the maximum in $R_{\mathrm{H}}(T)$, defined as $T_{\mathrm{m}}$, shifts to low temperature when approaching $H_{\mathrm{m}}$ down to $T_{\mathrm{CEP}}$. Above $H_{\mathrm{m}}$, the temperature of the maximum $T_{\mathrm{cr}}$, corresponding to the crossover temperature between the PM and PPM state, shifts to higher temperature. The energy scale associated with $T_{\mathrm{m}}$ (similar to $T_{\chi_{\max }}$ ) does not decrease to zero temperature but is limited to $T_{\mathrm{CEP}}$ due to the first-order nature of the transition and to the absence of a quantum critical point.

\section{Ordinary and anomalous Hall effect}

Here we describe the procedure to estimate the ordinary and anomalous contribution to the Hall effect. The first assumption is that the Hall signal is the sum of two terms, $R_{\mathrm{H}}=$ $R_{0}+R_{\mathrm{S}}$, where $R_{0}$ is the ordinary part associated with the density of carriers and their mobility and $R_{\mathrm{S}}$ is the anomalous part associated with scattering processes on magnetic impurities. The microscopic origin of $R_{\mathrm{S}}$ is quite complex. Three distinct contributions, intrinsic scattering, skew scattering, and side jump scattering have been identified. Each of them has an individual scaling $R_{\mathrm{S}} \propto \rho^{\alpha} M_{z} / H$ with respect to the longitudinal resistivity $\rho$. Here, $M_{z}$ is the magnetization and $H$ is the magnetic field along the $z$ axis. In ferromagnetic materials, the summation of the three terms yields an empirical formula that explains a large amount of experimental data. However, in heavy fermion materials a satisfactory formula has not been achieved, nevertheless it has been observed that the skew scattering is the dominant scattering process with two different scalings depending on the temperature [27,29]. At high temperature, for $T>T^{*}$ (coherence temperature), the incoherent skew scattering of conduction electrons by independent $f$ electrons should be considered, and then the relation $R_{\mathrm{S}}=C^{\prime} \times \rho M / H$ is expected. On the other hand, at low temperature for $T<T_{\mathrm{FL}}$ (Fermi liquid temperature) a different scaling is expected, $R_{\mathrm{S}}=C \times \rho^{2} M / H$, due to the coherent skew scattering of $f$ electrons once the Fermi 

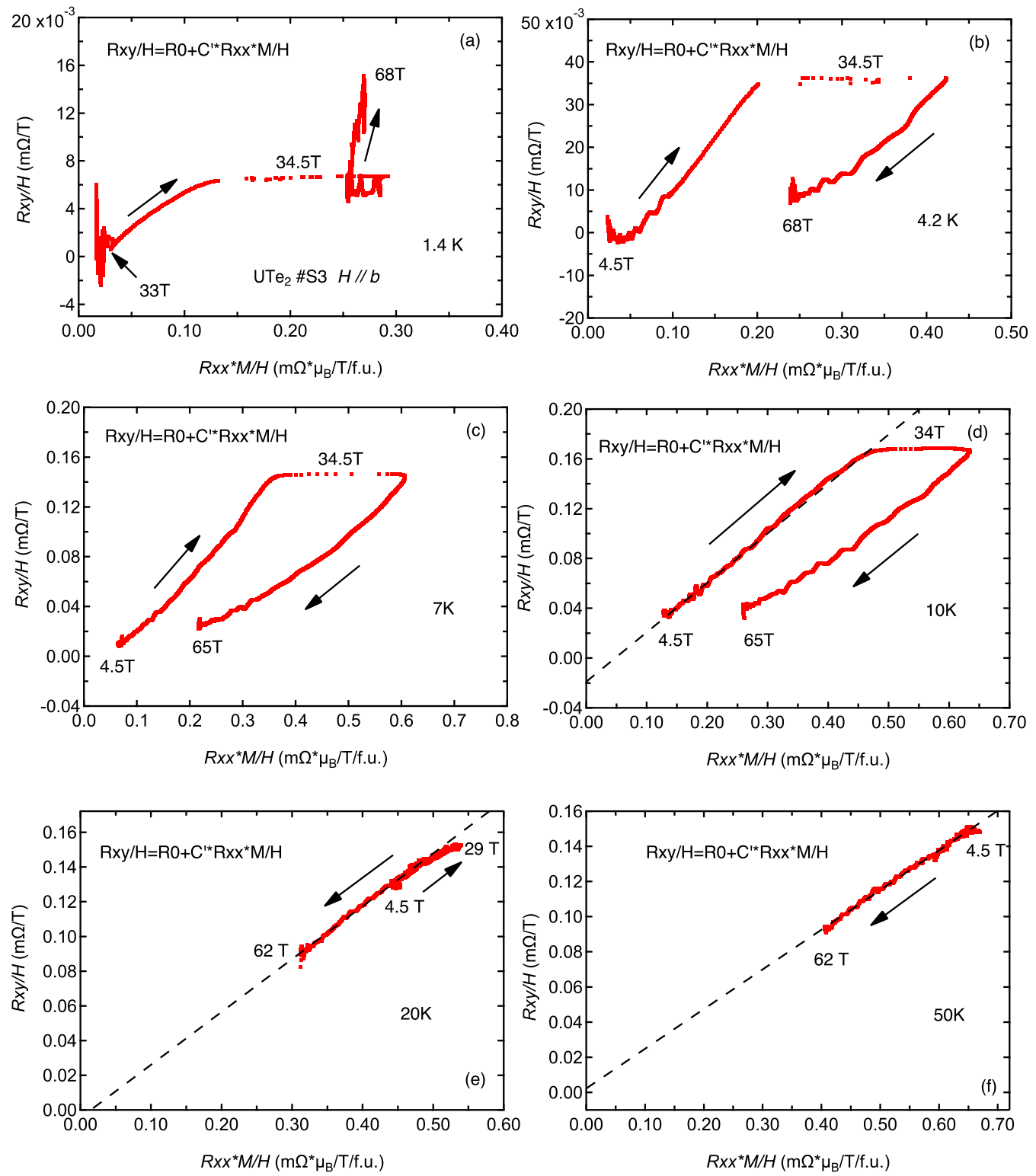

FIG. 11. $R_{x y} / H$ against $R_{x x} M / H$. The dashed lines are linear fits.

surface is well defined. Between $T^{*}$ and $T_{\mathrm{FL}}$ a crossover regime is observed. In Fig. 10, the temperature dependence of $R_{\mathrm{H}}$ is represented. The inset shows that as expected at low temperatures (for $T<20 \mathrm{~K}$ ), the Hall signal is well described by a linear fitting as a function of $\rho^{2} M / H$, indicating that below $T_{\chi_{\max }}$ a coherent regime appears. The fact that the fit $\left(R_{\mathrm{S} \_} \mathrm{LT}\right)$ is smaller than the $R_{\mathrm{H}}$ data at very low temperatures indicates that the anomalous contribution to the Hall signal is negligible at low temperature, and it is justified to extract the number of carriers by using the Hall signal above $T_{\mathrm{SC}}$ in this material [4]. The fit using $\rho M / H$ $\left(R_{\mathrm{S} \_} \mathrm{HT}\right)$ is not very satisfactory at high temperature. This discrepancy can be explained by the phonon contribution in the resistivity [29].
To extract the coefficients $C$ and $C^{\prime}$, we have plotted $R_{x y} / H$ as a function of $R_{x x} M / H$ in Fig. 11 and as a function of $R_{x x}^{2} M / H$ in Fig. 12. We observe that for $T<20 \mathrm{~K}$, the data are well described by $R_{x x}^{2} M / H$, meaning that below $T^{*}$ (roughly $T \chi_{\max }$ ), the anomalous Hall signal is well described by coherent skew scattering of $f$ electrons. For high temperatures, the anomalous Hall signal is well described by $R_{x x} M / H$ highlighting the predominance of incoherent skew scattering processes above $T^{*}$. These observations are summarized in Fig. 13 where $R_{x y} / H$ is plotted either as a function of $R_{x x} M / H$ or as a function of $R_{x x}^{2} M / H$ depending on the temperature. It is interesting to notice that for $T>20 \mathrm{~K}$, for the whole magnetic field range, the data are well fitted by a unique linear function of $R_{x x} M / H$ passing through the origin, meaning that 

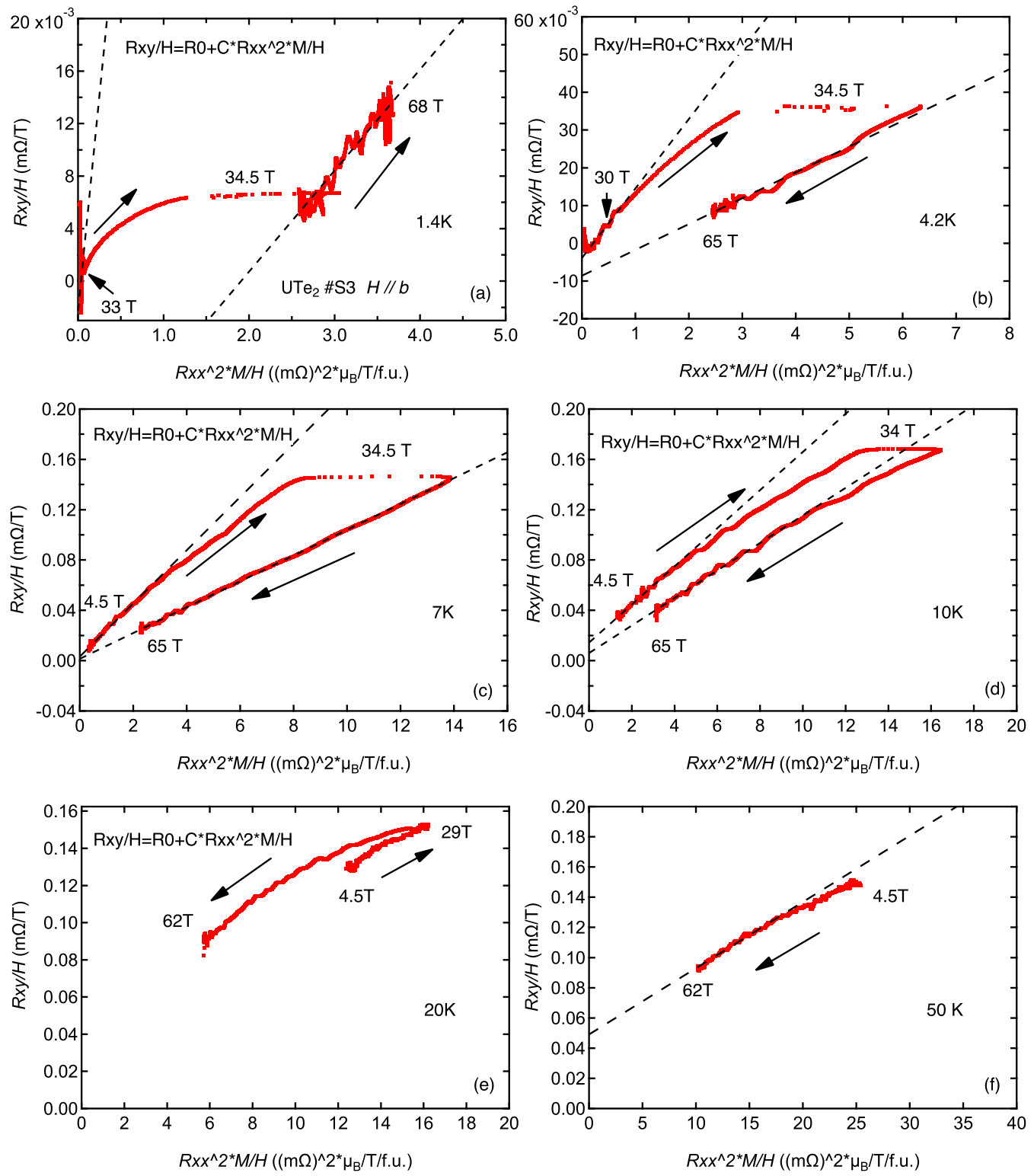

FIG. 12. $R_{x y} / H$ against $R_{x x}^{2} M / H$. The dashed lines are linear fits.

the ordinary Hall effect is negligible at high temperatures. At low temperatures, the data are clearly well described by two linear functions of $R_{x x}^{2} M / H$ below and above $H_{\mathrm{m}}$. The value of the coefficients $C$ and $C^{\prime}$ are summarized in Table I.

The variation of $C$ indicates that the amplitude of the scattering changes through $H_{\mathrm{m}}$. At $1.4 \mathrm{~K}$, below $H_{\mathrm{m}}$ the data do not show any linear dependence, meaning that the Hall signal is ordinary. On the other hand, above $H_{\mathrm{m}}$, the data still show linear dependence, meaning that even at very low temperatures, there is still a contribution from the anomalous
Hall effect above the metamagnetic transition just above $T_{\mathrm{SC}}$. After extracting the contribution of the anomalous Hall effect, through the different coefficients $C$ and $C^{\prime}$ depending on the temperature, we obtained the ordinary contribution by subtracting the anomalous part from the raw data by taking into account the change of the coefficient $C$ through $H_{\mathrm{m}}$. For all temperatures, the field dependence of the ordinary Hall effect is represented in Fig. 14. A drastic change of the ordinary Hall effect at $H_{\mathrm{m}}$ is extracted from this analysis, indicating a change of the number of carriers associated or not with a change of their mobility. 

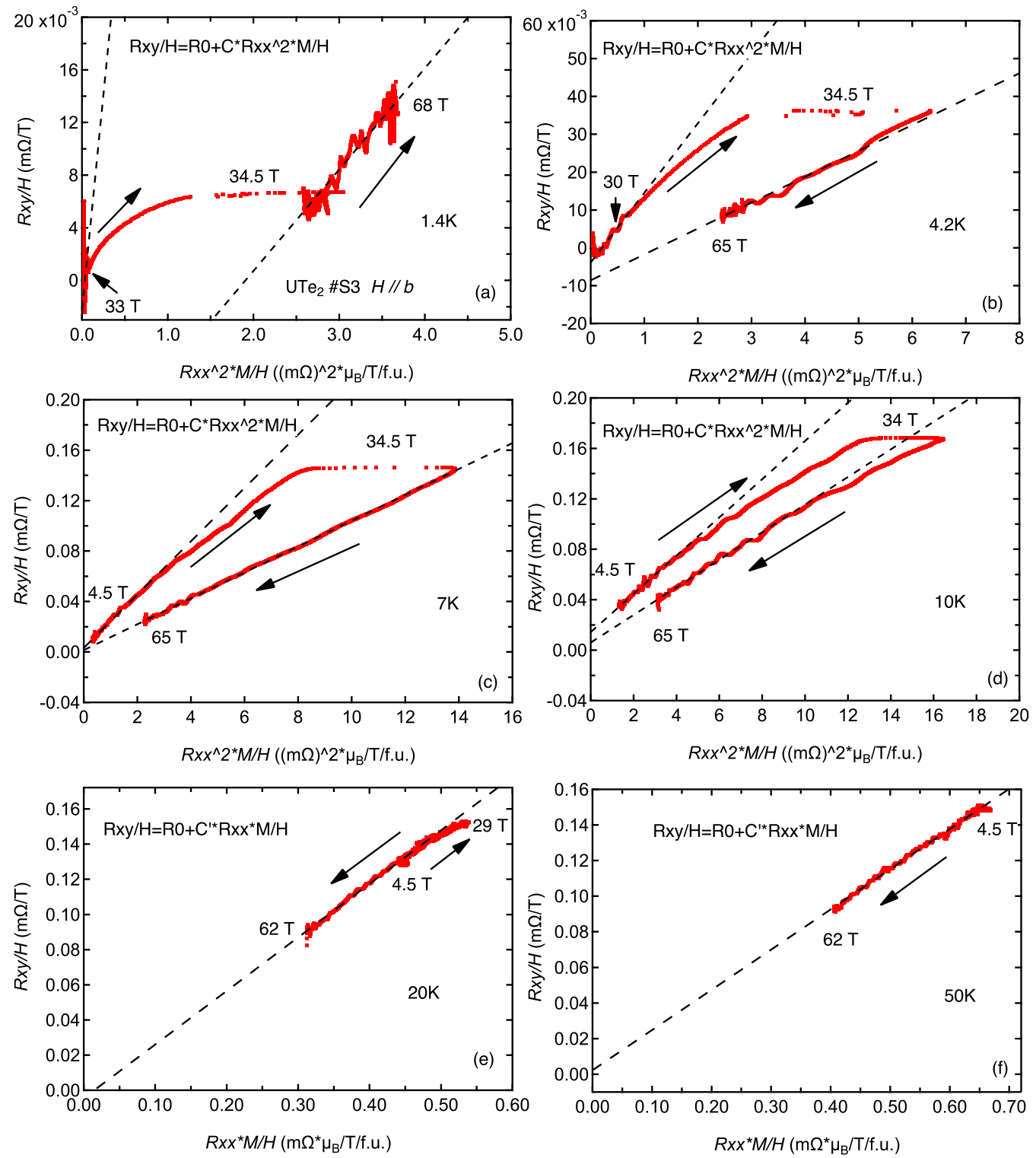

FIG. 13. $R_{x y} / H$ against $R_{x x}^{2} M / H$ at $1.4,4.2,7$, and $10 \mathrm{~K} . R_{x y} / H$ against $R_{x x} M / H$ at 20 and $50 \mathrm{~K}$. The dashed lines are linear fits.

TABLE I. List of anomalous Hall effect coefficients $C\left(R_{\mathrm{S}}=\right.$ $\left.C \times \rho^{2} M / H\right)$ for $H$ below and above $H_{\mathrm{m}}$ and $C^{\prime}\left(R_{\mathrm{S}}=C^{\prime} \times \rho M / H\right)$.

\begin{tabular}{lccc}
\hline \hline & \multicolumn{3}{c}{$C$ (arb. units) } \\
\cline { 2 - 3 }$T(\mathrm{~K})$ & $H<H_{\mathrm{m}}$ & $H>H_{\mathrm{m}}$ & $\frac{C\left(H>H_{\mathrm{m}}\right)}{C\left(H<H_{\mathrm{m}}\right)}$ \\
\hline 1.4 & 65.3 & 8.6 & 0.13 \\
4.2 & 18.4 & 7.6 & 0.41 \\
7 & 21.1 & 10.2 & 0.48 \\
10 & 13.9 & 10.5 & 0.75 \\
& \multicolumn{3}{c}{$C^{\prime}$ (arb. units) } \\
50 & \multicolumn{3}{c}{0.30} \\
\hline
\end{tabular}

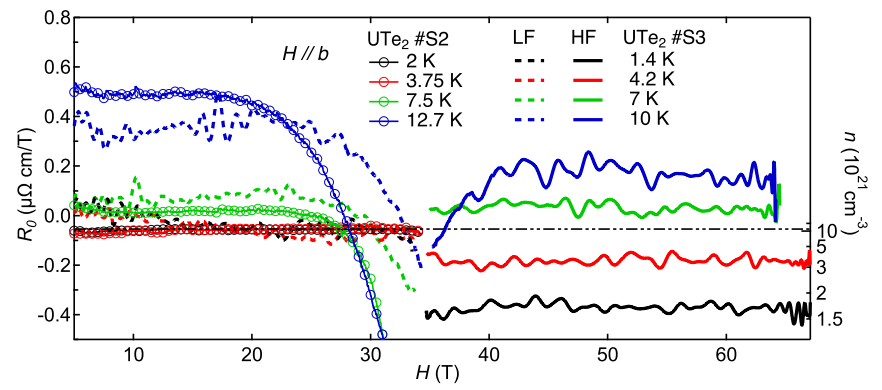

FIG. 14. Complement to Fig. 5(b) of the main text. Ordinary Hall effect as a function of the magnetic field for different temperatures obtained after subtracting the anomalous contribution (taking into account its change of amplitude through $H_{\mathrm{m}}$ ). This implies two sets of data for low field (dashed lines, LF) and high field (solid lines, $\mathrm{HF}$ ) for the sample $\mathrm{S} 3$ measured up to $68 \mathrm{~T}$. The right scale indicates the carrier density and the dashed-dotted line represents the value obtained previously [4]. 
[1] S. Ran, C. Eckberg, Q.-P. Ding, Y. Furukawa, T. Metz, S. R. Saha, I.-L. Liu, M. Zic, H. Kim, J. Paglione, and N. P. Butch, Science 365, 684 (2019).

[2] D. Aoki, A. Nakamura, F. Honda, D. Li, Y. Homma, Y. Shimizu, Y. J. Sato, G. Knebel, J.-P. Brison, A. Pourret, D. Braithwaite, G. Lapertot, Q. Niu, M. Vališka, H. Harima, and J. Flouquet, J. Phys. Soc. Jpn. 88, 043702 (2019).

[3] G. Knebel, W. Knafo, A. Pourret, Q. Niu, M. Vališka, D. Braithwaite, G. Lapertot, M. Nardone, A. Zitouni, S. Mishra, I. Sheikin, G. Seyfarth, J.-P. Brison, D. Aoki, and J. Flouquet, J. Phys. Soc. Jpn. 88, 063707 (2019).

[4] Q. Niu, G. Knebel, D. Braithwaite, D. Aoki, G. Lapertot, G. Seyfarth, J.-P. Brison, J. Flouquet, and A. Pourret, Phys. Rev. Lett. 124, 086601 (2020).

[5] J. Ishizuka, S. Sumita, A. Daido, and Y. Yanase, Phys. Rev. Lett. 123, 217001 (2019).

[6] S.-i. Fujimori, I. Kawasaki, Y. Takeda, H. Yamagami, A. Nakamura, Y. Homma, and D. Aoki, J. Phys. Soc. Jpn. 88, 103701 (2019).

[7] Y. Xu, Y. Sheng, and Y. F. Yang, Phys. Rev. Lett. 123, 217002 (2019).

[8] L. Miao, S. Liu, Y. Xu, E. C. Kotta, C.-J. Kang, S. Ran, J. Paglione, G. Kotliar, N. P. Butch, J. D. Denlinger, and L. A. Wray, Phys. Rev. Lett. 124, 076401 (2020).

[9] Y. Tokunaga, H. Sakai, S. Kambe, T. Hattori, N. Higa, G. Nakamine, S. Kitagawa, K. Ishida, A. Nakamura, Y. Shimizu, Y. Homma, D. Li, F. Honda, and D. Aoki, J. Phys. Soc. Jpn. 88, 073701 (2019).

[10] S. Ran, I.-L. Liu, Y. S. Eo, D. J. Campbell, P. M. Neves, W. T. Fuhrman, S. R. Saha, C. Eckberg, H. Kim, D. Graf, F. Balakirev, J. Singleton, J. Paglione, and N. P. Butch, Nat. Phys. 15, 1250 (2019).

[11] G. Nakamine, S. Kitagawa, K. Ishida, Y. Tokunaga, H. Sakai, S. Kambe, A. Nakamura, Y. Shimizu, Y. Homma, D. Li, F. Honda, and D. Aoki, J. Phys. Soc. Jpn. 88, 113703 (2019).

[12] A. Miyake, Y. Shimizu, Y. J. Sato, D. Li, A. Nakamura, Y. Homma, F. Honda, J. Flouquet, M. Tokunaga, and D. Aoki, J. Phys. Soc. Jpn. 88, 063706 (2019).

[13] S. Imajo, Y. Kohama, A. Miyake, C. Dong, M. Tokunaga, J. Flouquet, K. Kindo, and D. Aoki, J. Phys. Soc. Jpn. 88, 083705 (2019).
[14] W. Knafo, M. Vališka, D. Braithwaite, G. Lapertot, G. Knebel, A. Pourret, J.-P. Brison, J. Flouquet, and D. Aoki, J. Phys. Soc. Jpn. 88, 063705 (2019).

[15] D. Aoki, A. Huxley, E. Ressouche, D. Braithwaite, J. Flouquet, J. P. Brison, E. Lhotel, and C. Paulsen, Nature (London) 413, 613 (2001).

[16] A. Miyake, D. Aoki, and J. Flouquet, J. Phys. Soc. Jpn. 77, 094709 (2008).

[17] F. Hardy, D. Aoki, C. Meingast, P. Schweiss, P. Burger, H. v. Löhneysen, and J. Flouquet, Phys. Rev. B 83, 195107 (2011).

[18] B. Wu, G. Bastien, M. Taupin, C. Paulsen, L. Howald, D. Aoki, and J.-P. Brison, Nat. Commun. 8, 14480 (2017).

[19] A. Gourgout, A. Pourret, G. Knebel, D. Aoki, G. Seyfarth, and J. Flouquet, Phys. Rev. Lett. 117, 046401 (2016).

[20] D. Aoki, G. Knebel, and J. Flouquet, J. Phys. Soc. Jpn. 83, 094719 (2014).

[21] E. A. Yelland, J. M. Barraclough, W. Wang, K. V. Kamenev, and A. D. Huxley, Nat. Phys. 7, 890 (2011).

[22] Y. Sherkunov, A. V. Chubukov, and J. J. Betouras, Phys. Rev. Lett. 121, 097001 (2018)

[23] S. Sundar, S. Gheidi, K. Akintola, A. M. Côté, S. R. Dunsiger, S. Ran, N. P. Butch, S. R. Saha, J. Paglione, and J. E. Sonier, Phys. Rev. B 100, 140502(R) (2019).

[24] C. Paulsen, G. Knebel, G. Lapertot, D. Braithwaite, A. Pourret, D. Aoki, F. Hardy, J. Flouquet, and J. P. Brison, arXiv:2002.12724.

[25] J. Schoenes and J. J. M. Franse, Phys. Rev. B 33, 5138 (1986).

[26] M. Hadžić-Leroux, A. Hamzić, A. Fert, P. Haen, F. Lapierre, and O. Laborde, Europhys. Lett. 1, 579 (1986).

[27] Y.-F. Yang, Phys. Rev. B 87, 045102 (2013).

[28] A. Palacio Morales, A. Pourret, G. Seyfarth, M.-T. Suzuki, D. Braithwaite, G. Knebel, D. Aoki, and J. Flouquet, Phys. Rev. B 91, 245129 (2015).

[29] A. Fert and P. M. Levy, Phys. Rev. B 36, 1907 (1987).

[30] K. Yamada, K. Ani, H. Kohno, and S. Inagaki, Prog. Theor. Phys. 89, 1155 (1993)

[31] A. Palacio-Morales, A. Pourret, G. Knebel, T. Combier, D. Aoki, H. Harima, and J. Flouquet, Phys. Rev. Lett. 110, 116404 (2013). 